\title{
The majority of genes in the pathogenic Neisseria species are present in non-pathogenic Neisseria lactamica, including those designated as 'virulence genes' Lori AS Snyder* and Nigel J Saunders
}

Address: Bacterial Pathogenesis and Functional Genomics Group, Sir William Dunn School of Pathology, University of Oxford, Oxford, OX1 3RE, UK

Email: Lori AS Snyder* - lori.snyder@path.ox.ac.uk; Nigel J Saunders - nigel.saunders@path.ox.ac.uk

* Corresponding author

Published: 30 May 2006

BMC Genomics 2006, 7:128 doi:10.1186/147|-2164-7-128
Received: 0I February 2006

Accepted: 30 May 2006

This article is available from: http://www.biomedcentral.com/I47I-2/64/7//28

(c) 2006 Snyder and Saunders; licensee BioMed Central Ltd.

This is an Open Access article distributed under the terms of the Creative Commons Attribution License (http://creativecommons.org/licenses/by/2.0), which permits unrestricted use, distribution, and reproduction in any medium, provided the original work is properly cited.

\begin{abstract}
Background: Neisseria meningitidis causes the life-threatening diseases meningococcal meningitis and meningococcal septicemia. Neisseria gonorrhoeae is closely related to the meningococcus, but is the cause of the very different infection, gonorrhea. A number of genes have been implicated in the virulence of these related yet distinct pathogens, but the genes that define and differentiate the species and their behaviours have not been established. Further, a related species, Neisseria lactamica is not associated with either type of infection in normally healthy people, and lives as a harmless commensal. We have determined which of the genes so far identified in the genome sequences of the pathogens are also present in this non-pathogenic related species.

Results: Thirteen unrelated strains of $N$. lactamica were investigated using comparative genome hybridization to the pan-Neisseria microarray-v2, which contains 2845 unique gene probes. The presence of 127 'virulence genes' was specifically addressed; of these 85 are present in N. lactamica. Of the remaining 42 'virulence genes' only II are present in all four of the sequenced pathogenic Neisseria.

Conclusion: Assessment of the complete dataset revealed that the vast majority of genes present in the pathogens are also present in N. lactamica. Of the I,473 probes to genes shared by all four pathogenic genome sequences, I,373 hybridize to $N$. lactamica. These shared genes cannot include genes that are necessary and sufficient for the virulence of the pathogens, since N. lactamica does not share this behaviour. This provides an essential context for the interpretation of gene complement studies of the pathogens.
\end{abstract}

\section{Background}

Neisseria meningitidis and Neisseria gonorrhoeae are closely related bacterial pathogens, which share many common genes with a high degree of sequence identity (typically greater than 95\%). DNA relatedness studies cluster the commensal N. lactamica with the two pathogenic Neisseria spp. [1]. Sequence identity of housekeeping genes also clusters $N$. lactamica with the pathogens [2]. Unlike $N$. meningitidis and $N$. gonorrhoeae, the rare cases of disease caused by $N$. lactamica are largely due to some compromise in the patient, and it is not normally pathogenic [35]. 
The pan-Neisseria microarray-v2 was designed so that common probes are used to address all coding regions thus far identified in the pathogenic Neisseria spp., with 2716 probes to all of the probable coding regions from the four complete neisserial genomes, plus 129 probes for additional neisserial genes [6]. The probes were designed, based upon a comparative genomic analysis, to the most conserved regions within the genes, to optimize the microarray for use in investigations of strains against which it was not designed. Because the probes are minimally genome specific, being within the most conserved areas of the genes, it is possible to also use the pan-Neisseria microarray-v2 to assess the gene complement of the related non-pathogenic species $N$. lactamica.

The range of tools available to address the similarities, differences, and evolutionary relationships between bacterial species has been extended with the availability of multiple genome sequences from within related species groups and the development of microarray-based comparative genome hybridization studies. While traditional methods of comparison and typing are based upon the comparison of divergence within genes that are common and highly conserved, the genomic approach facilitates a very different and complementary method of investigation that allows the commonality and flux of genes, and combinations of genes, within and between species to be addressed. Methods such as MLEE and MLST broadly address genes that are considered to be essential and under minimal functional selection, and are thus under sufficiently low selective pressure to show changes over a useful period of evolutionary time for the purposes of a particular study. It is also the case, however, that horizontal exchange of genes and gene-systems is occurring that directly affect behaviour and these are frequently directly under selection. In two recent studies of another relatively panmictic species, Helicobacter pylori, findings relevant to this have recently been made. Firstly, it was shown that rather than being relatively immune to recombination, exchange is polarized in both "highly conserved" genes associated with central metabolic processes as well as those associated with strain-dependent behaviour. The former is driven by homology-dependent recombination and their highly conserved nature, and the latter by functional selection [7]. Secondly, it has been demonstrated that while differences in genes under selection broadly associate with the MLST-based population structure, clear examples of exchange occur between what the traditional relatedness trees consider to be very remote unrelated strains (for example between the hpAfrica2 population and hpAfrica1) (Salaün \& Saunders - unpublished data). This highlights the need for, and the complementary nature of, comparative genome hybridization studies to determine gene complements in studies of population structures and their evolution. This is because this approach measures a different comparatively slow type of change (the acquisition and loss of whole genes) that is potentially directly related to behavior. This may be key to determining which changes underlie the emergence and relative success of different clonal clusters, especially within relatively panmictic populations. In the context of studies of bacterial pathogens this approach has the potential to identify those genes or gene combinations that are responsible for the periodic increases and decreases in strains with differing pathogenic potentials.

Thirteen unrelated strains of $N$. lactamica were assessed through comparative genome hybridization to the panNeisseria microarray-v2 to determine which genes thought to be associated with virulence traits of the pathogenic Neisseria spp. are present in this non-pathogenic species. The pan-Neisseria microarray-v2 contains probes for the genes from the genome sequences of $N$. gonorrhoeae strain FA1090, N. meningitidis strain MC58, N. meningitidis strain Z2491, N. meningitidis strain FAM18, and to the genes from the $N$. gonorrhoeae strain MS11 Gonococcal Genetic Island, neisserial genes from GenBank/EMBL that did not yet have probes, and newly identified genes from Minimal Mobile Elements (see Additional files 2 \&3). While previously used subtractive methods [8] have been able to identify some of the genes missing from a small number of $N$. lactamica strains, hybridization to the microarray probes provides positive identification of the sequences that are present in this species.

\section{Results \\ Hybridization of N. lactamica to the pan-Neisseria microarray-v2}

The hybridization of the N. lactamica strains (Table 1) to a large proportion of the probes of the pan-Neisseria microarray-v2 is both a validation of the design strategy used for the microarray (see Additional file 3 ) and highlights the similarity of these species. This data can be easily visualized in its genomic context at http:// www.compbio.ox.ac.uk/data, where GBrowse databases detail the N. gonorrhoeae strain FA1090 and N. meningitidis strain MC58 and Z2491 genome sequences, their annotations, microarray probe locations and sequences, and indicate those probes that hybridize with $N$. lactamica in this study. In general, with $N$. lactamica neither the intensity of the hybridization signals nor the percentage of the microarray probes hybridized significantly differs from that seen in similar N. meningitidis or N. gonorrhoeae comparative genome hybridizations (Figure 1).

\section{N. lactamica hybridizes to 'virulence gene' probes}

A set of genes identified in the literature [8-10] to be involved in the virulence or pathogenesis of the Neisseria spp. (see Additional file 1) were specifically assessed through manual interpretation of the microarray image 
Table I: Strains used in this study.

\begin{tabular}{lll}
\hline Species & Strain & ST by MLST \\
\hline N. lactamica & $10102 \mathrm{M}$ & ST-613 \\
N. lactamica & 14804 & ST-595 \\
N. lactamica & $08802 S \mathrm{I}$ & ST-640 \\
N. lactamica & 908 & ST-598 \\
N. lactamica & 4116 & ST-615 \\
N. lactamica & 1520 & ST-592 \\
N. lactamica & 5804 & ST-616 \\
N. lactamica & 11004 & ST-1202 \\
N. lactamica & 224 & ST-624 \\
N. lactamica & 310 & ST-643 \\
N. lactamica & 12024 & ST-1205 \\
N. lactamica & 2510 & ST-609 \\
N. lactamica & Stephens & ND \\
\hline
\end{tabular}

data. The microarray scan images of each spot feature were individually analyzed to determine hybridization to the probes for this subset of genes. Most of these genes were identified in N. lactamica (Table 2). Of the 127 virulenceassociated genes assessed (of which 83 are present in all of the 4 pathogen genome sequences), 85 were present in at least one of the strains of $N$. lactamica. Many of the 42 remaining pathogen-specific genes are not present in all of the pathogenic Neisseria spp. genome sequences. Based upon microarray hybridizations, 11 of these 'virulenceassociated' genes that are universally present in the pathogen sequences did not produce hybridization signals from any of the N. lactamica strains tested (Table 3). Of these, analysis of the currently unpublished $N$. lactamica genome sequence of another strain of this species (The Wellcome Trust Sanger Institute - unpublished) indicates that divergent homologues of the pilus-associated genes pilC, pilD, pglD, and $p g l F$ are present in this sequenced strain of N. lactamica, as is a divergent version of the antigen-encoding $s s a 1$ gene. For the remaining 6 genes, the equivalent genomic region could be found in each case, yet these genes were absent, both here (Figure 2) and in the genome sequence as a whole. In the cases of $d c a, v i r G$, and the NMB1646 hemolysin gene, alternative genes are present in these locations (Figure 2, panels A, C, and E). These results suggest that of the total number of currently recognized 'virulence genes', excluding the influence of allelic differences, the presence of only six can now be considered to be pathogen-specific. Only two of these six genes, iga and $d c a$, have previously been reported to be pathogen-specific $[4,8,9,11-14]$.

\section{N. lactamica contains most of the genes in the pathogenic Neisseria spp., including some previously reported as pathogen-specific}

When the complete microarray dataset was interrogated, 2,137 probes hybridized to at least one of the strains of $N$. lactamica (see Additional file 2). Of these, 46 are strain

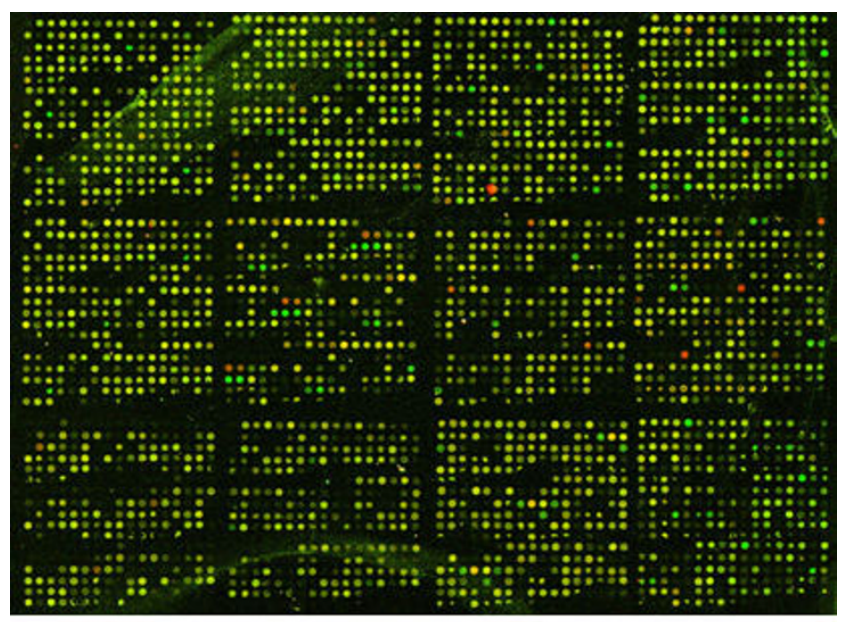

\section{N. meningitidis strains FAM18 and MC58}

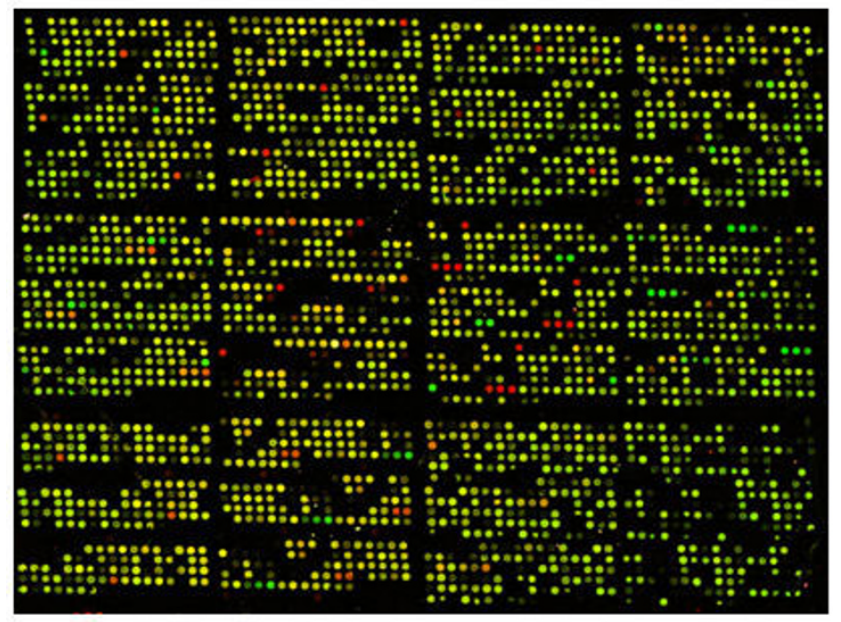

\section{N. lactamica strains}

\section{Figure I}

Hybridization to the pan-Neisseria microarray-v2.

specific, with the majority of these (33) being in the strain that was not part of the MLST set. The previously reported study of relatedness [1] of the neisserial species is probably heavily influenced by the genes that are shared between N. lactamica and the pathogenic Neisseria spp.

The N. lactamica strains used in this study possess several genes previously reported as pathogen-specific, including 
Table 2: Comparative genome hybridization to 'virulence gene' probes.

\begin{tabular}{|c|c|}
\hline Genes present in all of the strains of N. lactamica tested & Genes present in some of the strains of $N$. lactamica tested \\
\hline Surface proteins & Surface proteins \\
\hline opa, tspA, envA, rmpM, ompH, porB, NMB I946, mtrR, mtrD, mtrE, hsf, nlpD & tspB, $m$ trC, omp85, hap \\
\hline Iron acquisition & Iron acquisition \\
\hline tbp2, fbpA, bfrA, hemH, NMBI989-NMBI99I & IbpA, IbpB, bfrB, bcp, fbpB, tbpl, NMB2I32 \\
\hline Pilus associated & Pilus associated \\
\hline pgIE, pilG, pilF, pglH & pilE, pilT, NMB005I, pglA/pgtA, pg/B, pg/C, pg/G, pgll/wbpC \\
\hline LPS biosynthesis/regulation & LPS biosynthesis/regulation \\
\hline $\lg t A$, lgtB, lgtC, lgtD, lgtG, rfaC, rfaE, lpxB, IpxD, fabZ, pgm, phoQ/mis $S$ & rfaD, rfaF, rfaK, lgtF, IpxA, kdtA, phoP/misR, \\
\hline Others & Others \\
\hline $\begin{array}{l}\text { prc, hecB, hemagglutinin/hemolysins NMB0493 \& NMBI2I4, endonuclease } \\
\text { NMB0533, VapD-related NMBI753, macrophage infectivity potentiator } \\
\text { NMBI567, sialyltransferase NMB0922, adhesion NMB0586, TonB- } \\
\text { dependent receptor NMBI449, toxin-activating NMBI763 }\end{array}$ & $\begin{array}{l}\text { kat, dsbA, sodB, norZ, vapA, fur, vac/, hemagglutinin/ } \\
\text { hemolysin NMBI768, protease NMB2I 27, macrophage } \\
\text { infectivity potentiator NMB0995, toxin-activating } \\
\text { NMB I } 210 \text {, TonB-dependent receptors NMB } 346 \text { \& } \\
\text { NMBI829 }\end{array}$ \\
\hline
\end{tabular}

lipA, bioA, secY [9], mafA [15], and $d s b A[9,15]$. Of the 2,845 probes on the pan-Neisseria microarray-v2, 1,473 will hybridize to orthologues present in all four of the pathogenic sequenced strains, and the remainder addresses strain- or species- specific genes or highly divergent alleles. Of the 1,473 probes to genes that are universally present in the four pathogens' genome sequences, 1,373 hybridize with the N. lactamica strains tested.

\section{Comparison of our findings with those reported using the B $\mu \mathrm{G} @ S$ Neisseria microarray}

Following the design and public availability of the original pan-Neisseria microarray-v1 in 2002 (available from Prof. John Davies, Monash University, Australia) [16], the Bacterial Microarray Group at St. George's (B $\mu \mathrm{G} @ S$ ) designed a second, independent multi-strain Neisseria microarray that became available in 2004. During the time in which publication of this study was being pursued, the Bacterial Microarray Group published a study of the hybridization of several different Neisseria spp. to their microarray. Readers of other reports of neisserial microarray-based studies should note that the investigators at B $\mu \mathrm{G} @ S$ have also chosen to call their microarray the panNeisseria microarray, but this is not the same microarray used in our study. In their study, Stabler et al [17] hybridized two N. lactamica strains (L13 and L18) to their microarray. We have previously used these strains for other studies [18-20], but they were not included in the current study because they do not currently form part of a collection of known diversity and on their own do not represent a sufficient sample to address our experimental question related to $N$. lactamica. We had instead chosen to evaluate the gene complements of 12 MLST analyzed strains to assess the genes present in $N$. lactamica. To this data we added one other $N$. lactamica strain that we had used previously $[11,19]$. In addition, our study sought to identify genes that are present in $N$. lactamica, not those that are

Table 3: Pathogen-specific virulence genes absent in $\mathbf{N}$. lactamica.

\begin{tabular}{lll}
\hline No hybridization on microarraya & N. lactamica genome sequence results & Final results \\
\hline ssal & Present, but divergent & Present \\
pilC & Present, but divergent & Present \\
pilD & Present, possibly divergent in strains tested & Present \\
pgID & Present, but divergent & Present \\
pgIF & Present, possibly divergent in strains tested & Present \\
dcalpptA & Absent. The equivalent region contains an alternative hypothetical gene. & Absent \\
ABC transport (NMBI880) & Absent in genome sequence & Absent \\
TonB-dependent receptor, NMBI882 & Absent in genome sequence & Absent \\
virG & Absent. The equivalent region contains an alternative hypothetical gene. & Absent \\
iga encoding lgA protease & Absent in genome sequence & Absent \\
hemolysin, NMBI646 & Absent. The equivalent region contains alternative gene sequences. & Absent
\end{tabular}

aVirulence-associated genes that are present in all strains of the pathogenic Neisseria spp., but did not produce positive microarray hybridizations with N. lactamica.

bApproximately half of these genes are present in the unpublished genome sequence of $N$. lactamica, where sequence divergence from the pathogenic sequence can be observed. 

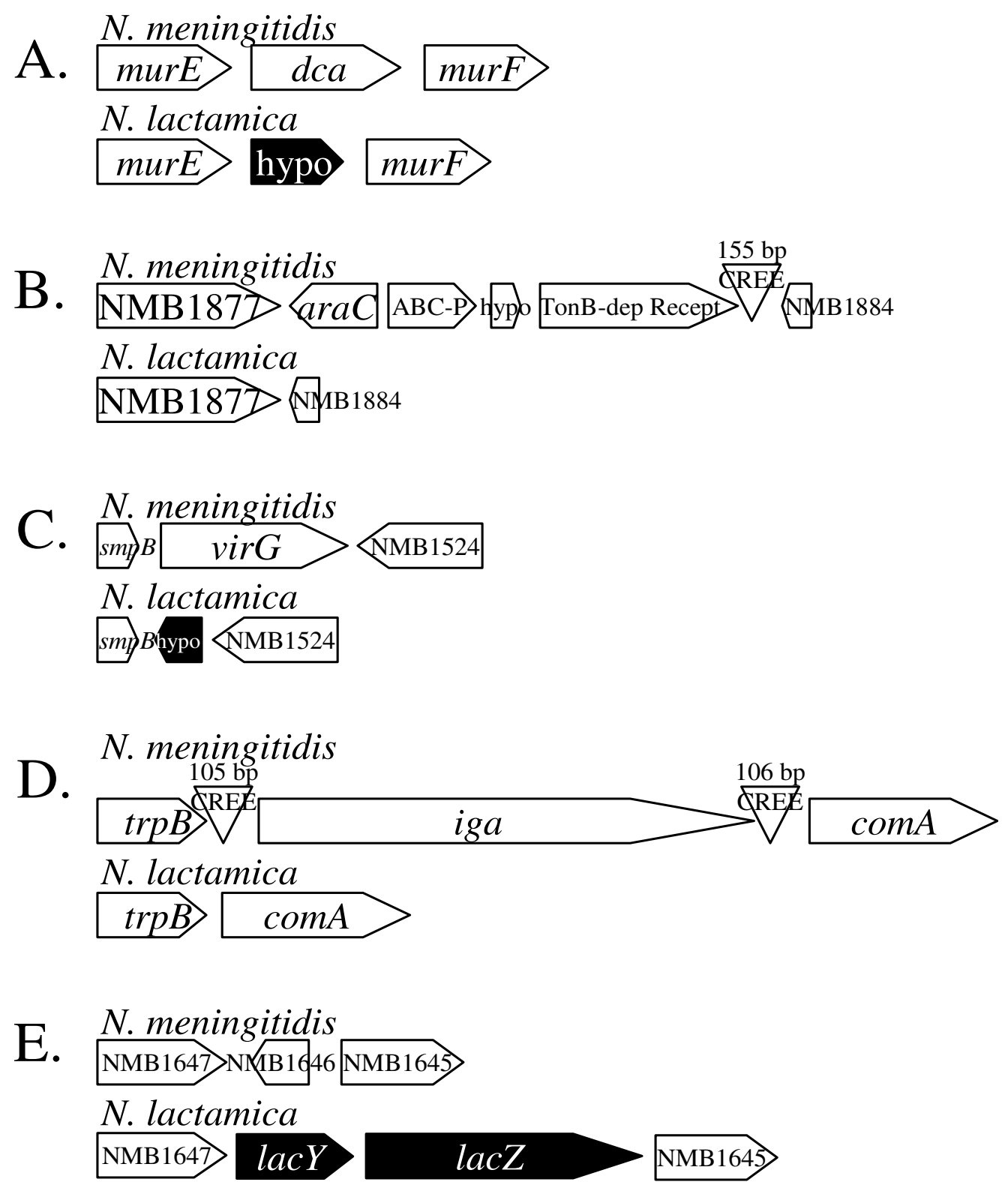

\section{Figure 2}

The chromosomal locations of the 6 'virulence genes' present in all pathogenic Neisseria spp. genome sequences, but absent from the $N$. lactamica genome sequence and for which no $N$. lactamica strains hybridized to the pan-Neisseria microarray-v2. Panel A: the region between murE and murF contains dca in the pathogen genome sequences and a hypothetical gene in the $N$. lactamica genome sequence. Panel B: the region between NMBI 877 and NMBI884 contains the 'virlence genes' NMBI880 (ABC-P, ABC transport periplasmic substrate-binding protein) and NMBI 882 (TonB-dependent receptor) as well as a hypothetical gene (NMBI88I) and an araC-like regulator in the pathogen genome sequences, which are all absent in this location in the $\mathrm{N}$. lactamica genome sequence. The probe for NMBI878, the araC regulator hybridized to all N. lactamica strains assessed by $\mathrm{CGH}$; this may be due to another araC-like regulator in another location or the presence of this regulator in this location in all the assessed strains. Panel C: the region between smpB and NMBI524 contains virG in the pathogen genome sequences and a hypothetical gene in the $N$. lactamica genome sequence. Panel D: the region between trpB and comA contains iga, flanked by Correia Repeat Enclosed Elements (CREE) commonly found in the neisserial genomes; iga and the CREEs are absent in the N. lactamica genome sequence. Panel E: the region between NMBI647 and NMBI 645 contains a putative hemolysin encoded by NMBI 646 in the pathogenic genome sequences, while in the N. lactamica genome sequence this is the location of lacY and lac Z, which confer the characteristic lactose fermentation phenotype of $N$. lactamica. 
absent from the commensal species, as was the goal of the Stabler et al [17] study.

Several of the genes reported by Stabler et al [17] as being absent from the commensal Neisseria spp., including their two strains of N. lactamica, are present in our N. lactamica hybridizations (Table 4). Whether these are due to differences in strain gene complement, microarray design, or data interpretation cannot be determined. In cases where the gene is present in only a few of our N. lactamica strains (see Additional file 2), the former may be the case, but where the gene probe hybridization is present in the majority of our strains, the later two points must be considered.

For purposes of comparing their findings with ours, the reported pilE and associated silent pils cassettes were not evaluated, since the possession of type II pili by N. lactamica [21] and pathogenic Neisseria strains such as N. meningitidis strain FAM18 [22] are well known and cannot be considered a differentiating factor between the pathogenic and commensal Neisseria spp. In addition, the results presented in Table 3 of absent genes in Stabler et al [17] includes separately listed copies of identical genes: NMA1167 and NMA1999 (100\% DNA ID; NMB0486/ NMB0970/NMB1741); and 9 reported copies of IS30. Discounting the additional copies of genes and the pilE/ pils sequences, 38 genes are reported to be absent in the commensal Neisseria spp. by Stabler et al [17]; in contrast, we find that 6 of these are present in our hybridizations with $N$. lactamica, and a further 14 produced positive hybridizations to our pan-Neisseria microarray-v2 with other commensal species (Table 4). This highlights the problematic nature of reporting an absence based on a lack of hybridization in microarray and similar hybridization-based studies, which is why our study was focused in the genes present in N. lactamica.

Of the remaining 18 genes reported by Stabler $e t$ al. that also did not give positive hybridization results in our study, four are within Minimal Mobile Elements (MMEs; NMB0415, NMB0468, NMB0470, and NMB1621), one (NMA0368) is lost upon the acquisition of XNG1985 to XNG1898, eight (NMB0230, NMB0304, NMB1737, NMB1738, NMA0510, NMA1218, NMA1219, and NMA1221) are associated with IS elements, and one is IS150. This is consistent with these being genuinely strain and species differentiating genes based upon other evidence, reflecting their strain-specific and mobile nature. In summary, of the 38 genes reported as absent in the commensals by Stabler $e t$ al., 20 are present in our hybridizations and 14 are probably genuine differences, but are associated with strain-specific mobile elements.

It is interesting to note that of the 6 pathogen-specific genes identified in our study (Table 3), iga, NMB1880,

Table 4: Genes reported as "absent from all commensal Neisseria species" by Stabler et al., 2005, which were positively hybridized in our commensal collection.

\begin{tabular}{|c|c|}
\hline \multicolumn{2}{|c|}{ Positive hybridizations with our N. lactamica collection: } \\
\hline \multicolumn{2}{|l|}{ NMB0239 } \\
\hline \multicolumn{2}{|l|}{ NMB0240 } \\
\hline \multicolumn{2}{|l|}{ NMB0294 } \\
\hline \multicolumn{2}{|l|}{ NMB0389 } \\
\hline \multicolumn{2}{|l|}{ NMB0832 } \\
\hline \multicolumn{2}{|l|}{ NMBI7I9 } \\
\hline \multicolumn{2}{|c|}{ Positive hybridizations in other commensal species: } \\
\hline NMB0083 & Neisseria elongata, Neisseria flavescens \\
\hline NMB0084 & Neisseria flavescens \\
\hline NMB0226 & Neisseria sicca \\
\hline NMB0227 & Neisseria sicca \\
\hline NMB0229 & Neisseria sicca \\
\hline NMB0293 & Neisseria elongata, Neisseria macacae, Neisseria sicca, Neisseria subflava \\
\hline NMB043I & Neisseria flavescens, Neisseria macacae, Neisseria sicca, Neisseria subflava \\
\hline NMB0435 & Neisseria flavescens, Neisseria macacae, Neisseria sicca, Neisseria subflava \\
\hline NMB0473 & Neisseria flavescens \\
\hline NMB0474 & Neisseria flavescens \\
\hline NMB0486* & Neisseria sicca \\
\hline NMB0654 & Neisseria polysaccharea, Neisseria sicca \\
\hline NMBI400 & Neisseria mucosa \\
\hline IS30 & Neisseria elongata, Neisseria macacae, Neisseria sicca, Neisseria subflava \\
\hline
\end{tabular}

* 100\% identical sequences are annotated as NMAI 167, NMA 1999, NMB0970, and NMBI74I. 
NMB1882, virG, and NMB1646 are not reported as absent by Stabler et al [17], however their absence in our hybridizations is consistent with their absence in the N. lactamica genome sequence (Figure 2). The apparent presence of IgA protease in their commensal Neisseria collection is particularly of note, however this is not discussed in their manuscript. In light of the previously established absence of IgA protease from N. lactamica [13], this observation must be assumed to be false positive data, either due to crosshybridization or data interpretation issues.

\section{Discussion}

In this study we were able to determine that 2,137 out of the 2,845 pan-Neisseria microarray-v2 probes hybridize in at least one strain of $N$. lactamica, that 85 of the 127 'virulence genes' are present in this non-pathogen, and that 1,373 of the 1,473 probes (93\%) to genes common to all of the four pathogenic Neisseria spp. genomes are present. The genomic locations of the hybridized probes can be visualized at http://www.compbio.ox.ac.uk/data, which presents this data in its genomic context relative to each of the published genome sequences. For the gonococcal database it also clearly relates the annotation used in this study, to that in GenBank (AE004969). The threshold for the interpretation of the microarray data was deliberately set to be highly stringent, so that a positive indication of gene presence could be reliably interpreted even in the context of the extension of this microarray to use on a species against which it was not designed. The data was then pooled, so that assay-to-assay variation in gene presence, hybridization, and divergence should not contribute to the overall gene presence results for the species.

Although probes on the pan-Neisseria microarray-v2 are designed to the most conserved regions of the genes from the pathogenic Neisseria spp., where multiple sequences of individual genes are available, substantially divergent sequences will not hybridize to the probes on the microarray. There can be differences (probably greater than at least 20\% divergence) between different allelic versions of genes that would lead to the lack of hybridization to the probes. Indeed, there are relatively weak hybridizations to some of the probes for genes that are known to have multiple divergent alleles or undergo antigenic variation, most notably to the various opa probes. The Sanger Institute's annotation and publication of the complete genome of a different strain of $N$. lactamica may reveal that some particularly divergent gene families that appear to be absent by microarray hybridization are actually present in $N$. lactamica, but are too divergent to be detected by our microarray probes (as in Table 3). It is therefore likely that some of the probes that did not hybridize will be revealed to be due to sequence divergence or other limitations of microarray methodology. However, such divergence is likely to be associated with functional differences between the encoded proteins. Likewise, once complete, the genome sequence of $N$. lactamica will identify genes that are not in the pathogenic Neisseria spp. strains studied to date and are not probed by the pan-Neisseria microarray-v2, such as the N. lactamica homologue of the Haemophilus influenzae licA, which is responsible for the presence of phosphorylcholine on the LPS of these commensal bacteria [23]. Such genome-wide analysis of the unpublished N. lactamica data is beyond the permissions given by the Sanger Institute for the use of their data before publication, therefore a genome-wide comparison of these results with the N. lactamica genome sequence has not been performed.

Due to the nature of microarray hybridizations, which are much like the interpretations of Southern blot data [24], only a positive hybridization can reliably be interpreted. The lack of hybridization to a probe spot is not interpretable data, since the negative result can arise from a number of factors that are not necessarily related to the absence of the gene. As can be seen in Table 3, the probes may not hybridize, although the gene sequence may be present. From a sequence standpoint, this can be due to divergence of the gene or absence of a portion of the gene that includes the gene probe. From a technology standpoint, some probes never function properly, due to secondary structures formed by the probe on the slide surface or the target in solution that prevent binding. Also, signal can be lost due to too little probe material, failure of the probe to transfer to the slide surface, pin failure in printing, variations in the slide surface, and variations in target labeling using random primers. Therefore, it is not possible to robustly interpret the lack of hybridization to the probes as a definitive indication of gene absence, which is why this study has focused on the identification of the genes present (rather than absent) in N. lactamica. If a reader so wishes, absent genes and regions can be easily inferred from the GBrowse databases, but the nature of this data should be carefully considered. Comparison of our data to the report of gene absence from the microarray hybridizations of Stabler et al [17] highlights the problematic nature of reporting negative hybridization results. Additionally, the genes with positive hybridizations indicate only that the sequence is present and cannot rule out frame-shifts or other inactivating mutations of these genes, which may become apparent from the N. lactamica genome sequence upon its completion. An investigation of the regions specific to the pathogens has already been conducted [8], which this study complements.

The behaviors of $N$. gonorrhoeae, N. meningitidis, and $N$. lactamica are distinct and clearly differentiate these three species. In other bacterial species, the differentiation between pathogenic and non-pathogenic related bacteria has frequently been associated with the acquisition/pres- 
ence of key 'virulence genes'. For example, the CTX prophage [25] and VPI [26] in Vibrio cholera, which encode cholera-toxin and toxin coregulated pilus; the LEE [27] in E. coli responsible for the attaching and effacing effects in EPEC and EHEC strains; and the species-defining SPI1 and SPI2 of Salmonella [28,29], which encode type III secretion systems. In the Neisseria spp., however, no such clear-cut event in the evolutionary history of these organisms has been identified to-date to account for the behavioural differences between these species, although the presence of the capsule locus is usually associated with disease-causing isolates of $N$. meningitidis. From the results presented here, it would appear that the events which lead to the evolution of the behaviors of the three closely related $N$. gonorrhoeae, N. meningitidis, and $N$. lactamica species have been far more subtle than in other pathogenic species groups, and that the basis for their behavioral differences may not be defined by a single event, a single set of genes, or loss of a single function. Instead, a complex integrated network of genes, regulation, and diversity of function in common genes may be responsible. For example, there are links between the ability to phase vary the pgtA gene in $N$. gonorrhoeae and the organism's ability to cause disseminated disease [30]. Thus, two strains of $N$. gonorrhoeae may be nearly identical, but due to a few polymorphisms in the repeat tract of pgtA they may have different disease potentials. As an example of differential regulation between the pathogenic species, the expression of the Mtr efflux pump, which is important for $N$. gonorrhoeae survival in the female genital tract [31], is not regulated by MtrR in N. meningitidis [32]. In addition, we have found that the MarR regulator controls different genes in the two pathogenic Neisseria spp. (Snyder \& Saunders - unpublished data). The genetic similarities and differences between the pathogens and $N$. lactamica identified in this study provide an additional framework from which to pursue these issues.

From a practical perspective, because natural immunity can follow colonization with $N$. lactamica, it can be argued that an ideal vaccine would protect against both pathogenic species but not against $N$. lactamica. However, this study shows that the number of such candidates is very limited. Modeling of meningococcal and N. lactamica carriage shows that colonization with the commensal provides some immunological protection against the pathogen. Vaccination against something common to $\mathrm{N}$. lactamica and the pathogens that reduces the carriage of $N$. lactamica is predicted to negatively impact the effectiveness of immunization programs [33]. This study shows that there may be as few as 100 potential candidates for such a vaccine (1,373 of 1,473 common pathogenic Neisseria probes are hybridized by $N$. lactamica), only a portion of which are likely to be suitable immunological targets.

\section{Conclusion}

Most of the genes that are considered to be virulence genes in the pathogenic Neisseria spp. are actually present within strains of $N$. lactamica, which is not pathogenic. The differences between these species, which make them pathogenic or not, are therefore not as great as might be supposed on the basis of their distinct behaviors and previous lists of virulence genes. Aside from genes such as the capsule biosynthesis genes, which are also not present in pathogenic $N$. gonorrhoeae, N. lactamica seems to only be missing a few of the genes that have been considered todate as virulence genes. The similarity between the gene complements of the commensals and pathogens suggests that the virulence of the pathogenic Neisseria spp. may not lie within the genes they possess per se, but rather in a 'genetic personality' which is a result of the combinations of these genes, sequence variations that alter the function of gene products, the possession of genes for which a virulence phenotype has not yet been identified, and/or in differences in the regulation of genes between the species. We have recently shown that in $N$. gonorrhoeae the ability to cause disseminated gonococcal infections is dependent upon the specific combinations of genes present, rather than being due to a 'virulence' or 'invasion' gene [34]. The same may be true in the wider context of pathogenic potential and commensal colonization when $N$. meningitidis and N. gonorrhoeae are compared with N. lactamica. Without the specific combination of pathogenic-potential genes, $N$. lactamica can possess the 'virulence genes' of the pathogens, yet remain a harmless commensal.

\section{Methods \\ Bacterial strains}

Thirteen strains of N. lactamica (Table 1) were investigated for positive hybridization to gene probes on the pan-Neisseria microarray-v2. Twelve of the strains were provided by Julia Bennett and Martin Maiden from their MLST study [35] and were chosen by them as representative strains of the population structure of this species. Additional information on these strains, or representative strains from these STs, can be obtained from the Neisseria MLST Home Page [41]. A thirteenth strain of N. lactamica was obtained previously from David Stephens. This strain has been used by us in previous studies $[19,20]$ and was added to this study to determine if additional data could be obtained from this strain. Eight commensal Neisseria spp. were investigated for positive hybridization to probes for genes previously reported by Stabler $e$ t al [17] as producing negative hybridizations in their study using the B $\mathrm{GG} @ \mathrm{~S}$ neisserial microarray. Neisseria cinerea, Neisseria elongata, Neisseria flavescens, Neisseria macacae, Neisseria mucosa, Neisseria polysaccharea, Neisseria sicca, and Neisseria subflava were obtained from Prof. David Stephens (Emory University), which have been used in previous studies $[11,19]$. 


\section{The pan-Neisseria microarray-v2}

The expanded pan-Neisseria microarray-v2 [6] was designed and generated using the same strategy as described previously for the pan-Neisseria microarray-v1 [16]. The microarray includes probes to the genome sequences of $N$. gonorrhoeae strain FA1090 (AE004969), N. meningitidis strains MC58 [10], Z2491 [36], and FAM18 (The Wellcome Trust Sanger Institute - unpublished), plus the N. gonorrhoeae strain MS11 Gonococcal Genetic Island [37], neisserial genes from GenBank/EMBL that are not in any of the genome sequences currently available (see Additional file 2 for GenBank Accessions), genes identified through our on-going investigations of MMEs $[16,19]$ (see Additional file 2 for GenBank Accessions), and antibiotic resistance cassette markers commonly used in mutagenesis. Each probe of 150-450 bp targets the most conserved ( $\geq 90 \%$ identical over at least 150 bp) non-repetitive region within each coding sequence, maximizing hybridization with common probes to orthologous genes in different strains and species. Any significant cross-hybridization was eliminated, where possible, or noted, thereby minimizing the potential for cross-hybridization with other genes, which would generate false positive results. At the time this microarray was designed and constructed there was no publicly available annotation for the N. gonorrhoeae strain FA1090 genome sequence, therefore the microarray probes were designed using our own annotation of this sequence. This is available to view against the genome sequence, with comparisons to the other annotations, showing the probe sequences, locations, and those hybridized by N. lactamica at http://www.compbio.ox.ac.uk/data. For a more detailed description of the probe design and availability of the pan-Neisseria microarray, see Additional file 3. The PCR product probes generated were suspended in Genetix Spotting Solution for Amine slides and printed with nonadjacent replicates in triplicate onto Genetix Amine microarray slides using a Genetix QArray Mini microarray printer with $150 \mu \mathrm{m}$ aQu solid pins.

\section{Comparative genome hybridization}

Chromosomal DNA was extracted using a phenol-based method [38] from the strains listed in Table 1, following growth on GC media (BD) with Kellogg and ferric nitrate supplements [39] at $37^{\circ} \mathrm{C}, 5 \% \mathrm{CO}_{2}(\mathrm{v} / \mathrm{v})$ overnight.

$20 \mu \mathrm{g}$ of DNA was fluorescently labeled through direct incorporation of FluoroLink ${ }^{\mathrm{TM}}$ Cy3-dCTP or FluoroLink ${ }^{\mathrm{TM}}$ Cy5-dCTP (Amersham Pharmacia Biotech) using 5 units of DNA polymerase I, Klenow fragment (Bioline) and $3 \mu \mathrm{g}$ random hexamer primers (Invitrogen) in a $50 \mu \mathrm{l}$ reaction for 1.5 to 4 hours at $37^{\circ} \mathrm{C}$. Unincorporated nucleotides and random primers were removed using QIAquick Nucleotide Removal columns (QIAGEN) according to the manufacturer's instructions.
Hybridizations were conducted in $4 \times$ SSC, $0.3 \%$ SDS under LifterSlips (Erie Scientific) at $65^{\circ} \mathrm{C}$ overnight. The slides were washed in $65^{\circ} \mathrm{C} 1 \times \mathrm{SSC}, 0.05 \%$ SDS for $2 \mathrm{~min}$ utes, $0.06 \times$ SSC for 2 minutes, and fresh $0.06 \times$ SSC for 2 minutes before drying using an airbrush. Microarray slides were scanned using a ScanArray Express HT (Perkin Elmer).

Thirteen $N$. lactamica strains were assessed, twelve of which were selected for their diversity based on previous MLST analysis [40]. The thirteenth strain was provided from the collection of Prof. David Stephens (Emory University). Each strain of N. lactamica was hybridized to two slides, was paired with different strains in each hybridization, and was labeled with Cy3 on one slide and Cy5 on the other. This generated triads of strains and slides; for example strain 'A'-Cy3 was hybridized with strain 'B'-Cy5, strain 'B'-Cy3 with strain 'C'-Cy5, and strain 'C'-Cy3 with strain 'A'-Cy5.

\section{Microarray data analysis using BlueFuse}

Microarray spot features were identified, quantified, and assessed using BlueFuse for Microarrays v2 (BlueGnome). Initially, all of the probes for a set of 127 previously described virulence genes for the pathogenic Neisseria (see Additional file 1) were visually checked for hybridization for each strain on both slides.

The complete dataset was then addressed using the BlueFuse for Microarrays (BlueGnome) pON score. The pON score non-comparatively evaluates the data from each microarray spot and uses this to report a probability of there being a hybridization signal for each spot. Unlike ratio-metric methods, this score is not dependent upon or influenced by the signal in the other channel. A pON score of zero indicates that there is no evidence for spot hybridization, while a score of one indicates that there is strong evidence that the spot has hybridized. The pON score is quantitatively calculated from two features of the data: the intensity of signal above background, and the circularity and uniformity that is characteristic of a microarray spot. First, the spot is evaluated to determine the proportion of pixels within it that are statistically inconsistent with the Bayesian calculated background noise, thus the amount of the spot that is determined to be hybridization signal. Next, since a simple signal score can be influenced by inconsistent background and other features that are not hybridized microarray spots, the characteristics of the spot feature are taken into account, i.e. whether it is round and the signal across it is uniform. This serves to discriminate between signals that are simply large, high intensity noise as opposed to those which are consistent with a hybridized microarray spot. The exact details of the calculation of this score are proprietary to BlueGnome. In CGH applications, it therefore provides 
an independent assessment of gene presence or absence in each channel. Each probe was printed onto the pan-Neisseria microarray slide in three non-adjacent locations, therefore three $\mathrm{pON}$ scores were available for each probe. The images were manually flagged within BlueFuse to remove all spots that were generating clearly artefactual data (such as due to dust and local hybridization effects), and the data removed from subsequent analysis. Regions of the slide were manually assessed to determine the range of pON scores that could consistently be relied upon to reflect a strong positive hybridization. Additionally, the pON scores were determined for control hybridizations to the pan-Neisseria microarray-v2 with the strains against which the probes were designed. For this study, the pON score for a positive probe hybridization was purposely selected to be highly stringent, at $\mathrm{pON}>0.67$, so that the genes called as present within $N$. lactamica could be relied upon to be present in this species; a lower pON score might reflect hybridization of probes to divergent alleles and orthologous genes of different function. The control hybridizations suggest that with the pathogenic Neisseria spp. pON scores $>0.49$ are reliable, therefore the high threshold for this study should be very robust. When all three probe spot replicates had pON scores $>0.67$ then the presence of that gene within the N. lactamica genome was accepted without further review of the slide image. For any other probes with pON scores of $>0.67$ for one or two spots, the slide image was reviewed to ascertain whether hybridization was present, indicating gene presence in the strain. The results were then combined for all $13 \mathrm{~N}$. lactamica strains. The data reported here is therefore from a pool of data that minimizes strain-specific influences on the data, either due to the absence of certain sequences in certain strains or the presence of differences within divergent gene families between strains. In all, with 8,535 probe spots on each microarray (2845 probes printed in triplicate), 17,070 probe spots were assessed per strain, for a total of 221,910 features that were evaluated in the whole study. This does not include the data for the other commensal Neisseria spp. hybridizations. The images for these were manually assessed for the presence of positive hybridization in the 32 gene probes that produced negative hybridizations in both our analysis of $N$. lactamica and that of Stabler et al [17].

\section{Authors' contributions}

LS conducted the microarray hybridizations, analysed the microarray data, wrote the manuscript, assisted in probe design for the pan-Neisseria microarray, and produced the pan-Neisseria microarray-v2 PCR product probes. NS conceived of the pan-Neisseria microarray and its use in this kind of study, was the primary designer of the probes for the microarray, manufactured the pan-Neisseria microarray-v2 slides used in this study, supervised this work, and provided support in the preparation of the manu- script. All authors read and approved the final manuscript.

\section{Additional material}

\begin{abstract}
Additional file 1
Virulence-associated and predicted virulence-associated genes from the pathogenic Neisseria spp. This file contains a table of those genes from the current published literature that have been suggested to have a role in virulence. Genes for which there were positive hybridizations on the panNeisseria microarray- $v 2$ in the $\mathrm{N}$. lactamica strains tested are indicated, as are those genes present in all four of the currently available Neisseria spp. genome sequences.

Click here for file

[http://www.biomedcentral.com/content/supplementary/14712164-7-128-S1.pdf]
\end{abstract}

\section{Additional file 2}

Genes from the pathogenic Neisseria spp. present in one or more of $13 \mathrm{~N}$. lactamica strains. This file contains a table of all of those genes for which the associated pan-Neisseria microarray-v2 probe was hybridized by at least one of the $\mathrm{N}$. lactamica strains tested. The number of strains with positive hybridizations is indicated.

Click here for file

[http://www.biomedcentral.com/content/supplementary/14712164-7-128-S2.pdf]

\section{Additional file 3}

Probe content and design for the pan-Neisseria microarray, versions 1 \& 2. This file provides a detailed description of the design and construction of the pan-Neisseria microarray and the revised and updated pan-Neisseria microarray-v2. Included are details of the probe design and selection criteria, the sequences against which the microarray was designed, additions to the microarray in version 2 , and publications that have used the pan-Neisseria microarray in CGH.

Click here for file

[http://www.biomedcentral.com/content/supplementary/14712164-7-128-S3.pdf]

\section{Acknowledgements}

LASS and this work were supported by a Wellcome Trust project grant awarded to NJS. The MLST N. lactamica strains were provided by Martin Maiden and Julia Bennett. Simon McGowan of the Sir William Dunn School of Pathology/Weatherall Institute of Molecular Medicine Computational Biology Research Group established the databases at http://www.comp bio.ox.ac.uk/data and provided bioinformatics support for this project. This work made use of sequence data produced by the Neisseria lactamica Sequencing Group at the Sanger Institute and can be obtained from ftp:// ftp.sanger.ac.uk/pub/pathogens/nl/. This work made use of sequence data produced by the Neisseria meningitidis serogroup C strain FAMI 8 Sequencing Group at the Sanger Institute and can be obtained from ftp://

ftp.sanger.ac.uk/pub/pathogens/nm/. The $N$. gonorrhoeae genome sequence was obtained from the University of Oklahoma, the Gonococcal Genome Sequencing Project which was supported by USPHS/NIH grant \#AI-38399, GenBank accession number AE004969.

\section{References}

I. Guibourdenche M, Popoff MY, Riou JY: Deoxyribonucleic acid relatedness among Neisseria gonorrhoeae, $N$. meningitidis, 
N. lactamica, N. cinerea and "Neisseria polysaccharea". Ann Inst Pasteur Microbiol 1986, I37B:I77-I85.

2. Smith NH, Holmes EC, Donovan GM, Carpenter GA, Spratt BG: Networks and groups within the genus Neisseria: analysis of argF, recA, rho, and I6S rRNA sequences from human Neisseria species. Mol Biol Evol 1999, 16:773-783.

3. Wilson HD, Overman TL: Septicemia due to Neisseria lactamica. J Clin Microbiol 1976, 4:2 I4-215.

4. Schifman RB, Ryan KJ: Neisseria lactamica septicemia in an immunocompromised patient. J Clin Microbiol 1983, 17:934-935.

5. Denning DW, Gill SS: Neisseria lactamica meningitis following skull trauma. Rev Infect Dis 1991, 13:216-218.

6. Snyder LAS, Jarvis SA, Saunders NJ: Complete and variant forms of the 'Gonococcal Genetic Island' in Neisseria meningitidis. Microbiology 2005, I 5 I:4005-40 I3.

7. Saunders NJ, Boonmee P, Peden JF, Jarvis SA: Inter-species horizontal transfer resulting in core-genome and niche-adaptive variation within Helicobacter pylori. BMC Genomics 2005, 6:9.

8. Perrin $A$, Nassif $X$, Tinsley $C$ : Identification of regions of the chromosome of Neisseria meningitidis and Neisseria gonorrhoeae which are specific to the pathogenic Neisseria species. Infect Immun 1999, 67:6119-6129.

9. Perrin A, Bonacorsi S, Carbonnelle E, Talibi D, Dessen P, Nassif $X$ Tinsley $C$ : Comparative genomics identifies the genetic islands that distinguish Neisseria meningitidis, the agent of cerebrospinal meningitis, from other Neisseria species. Infect Immun 2002, 70:7063-7072.

10. Tettelin H, Saunders NJ, Heidelberg J, Jeffries AC, Nelson KE, Eisen JA, Ketchum KA, Hood DW, Peden JF, Dodson RJ, Nelson WC, Gwinn ML, DeBoy R, Peterson JD, Hickey EK, Haft DH, Salzberg SL, White O, Fleischmann RD, Dougherty BA, Mason T, Ciecko A, Parksey DS, Blair E, Cittone H, Clark EB, Cotton MD, Utterback TR, Khouri H, Qin H, Vamathevan J, Gill J, Scarlato V, Masignani V, Pizza M, Grandi G, Sun L, Smith HO, Fraser CM, Moxon ER, Rappuoli R, Venter JC: Complete genome sequence of Neisseria meningitidis serogroup B strain MC58. Science 2000, 287: |809-18|5.

II. Snyder LAS, Saunders N], Shafer WM: A putatively phase variable gene (dca) required for natural competence in Neisseria gonorrhoeae but not Neisseria meningitidis is located within the division cell wall (dcw) gene cluster. J Bacteriol 200I, 183:1233-124|.

12. Wolff $K$, Stern $A$ : Identification and characterization of specific sequences encoding pathogenicity associated proteins in the genome of commensal Neisseria species. FEMS Microbiol Lett 1995, I 25:255-263.

13. Muller HE: Lack of immunoglobulin A protease in Neisseria lactamica. Eur J Clin Microbiol I 983, 2:153-I54.

14. Jose J, Otto GW, Meyer TF: The integration site of the iga gene in commensal Neisseria sp. Mol Genet Genomics 2003, 269:197-204.

15. Grifantini R, Bartolini E, Muzzi A, Draghi M, Frigimelica E, Berger J Randazzo F, Grandi G: Gene expression profile in Neisseria meningitidis and Neisseria lactamica upon host-cell contact: from basic research to vaccine development. Ann N Y Acad Sc 2002, 975:202-216.

16. Snyder LAS, Davies JK, Saunders NJ: Microarray genomotyping of key experimental strains of Neisseria gonorrhoeae reveals gene complement diversity and five new neisserial genes associated with Minimal Mobile Elements. BMC Genomics 2004 , 5:23

17. Stabler RA, Marsden GL, Witney AA, Li Y, Bentley SD, Tang CM, Hinds J: Identification of pathogen-specific genes through microarray analysis of pathogenic and commensal Neisseria species. Microbiology 2005, 1 51:2907-2922.

18. Jordan P, Snyder LA, Saunders NJ: Diversity in coding tandem repeats in related Neisseria spp. BMC Microbiol 2003, 3:23.

19. Saunders NJ, Snyder LA: The minimal mobile element. Microbiology 2002, 148:3756-3760.

20. Snyder LAS, Shafer WM, Saunders NJ: Divergence and transcriptional analysis of the division cell wall (dcw) gene cluster in Neisseria spp. Mol Microbiol 2003, 47:43।-442.

21. Aho EL, Keating AM, McGillivray SM: A comparative analysis of pilin genes from pathogenic and nonpathogenic Neisseria species. Microb Pathog 2000, 28:8I-88.
22. Aho EL, Cannon JG: Characterization of a silent pilin gene locus from Neisseria meningitidis strain FAM 18. Microb Pathog 1988, 5:391-398.

23. Serino L, Virji M: Phosphorylcholine decoration of lipopolysaccharide differentiates commensal Neisseriae from pathogenic strains: identification of licA-type genes in commensal Neisseriae. Mol Microbiol 2000, 35:1550-1559.

24. Southern EM: Detection of specific sequences among DNA fragments separated by gel electrophoresis. J Mol Biol 1975, 98:503-517.

25. Waldor MK, Mekalanos Jj: Lysogenic conversion by a filamentous phage encoding cholera toxin. Science 1996, 272:1910-1914

26. Karaolis DK, Johnson JA, Bailey CC, Boedeker EC, Kaper JB, Reeves PR: A Vibrio cholerae pathogenicity island associated with epidemic and pandemic strains. Proc Natl Acad Sci U S A 1998 , 95:3134-3139.

27. McDaniel TK, Jarvis KG, Donnenberg MS, Kaper JB: A genetic locus of enterocyte effacement conserved among diverse enterobacterial pathogens. Proc Natl Acad Sci U S A 1995, 92: 1664-I668.

28. Hensel M, Shea JE, Gleeson C, Jones MD, Dalton E, Holden DW: Simultaneous identification of bacterial virulence genes by negative selection. Science 1995, 269:400-403.

29. Shea JE, Hensel M, Gleeson C, Holden DW: Identification of a virulence locus encoding a second type III secretion system in Salmonella typhimurium. Proc Natl Acad Sci U S A 1996, 93:2593-2597.

30. Banerjee A, Wang R, Supernavage SL, Ghosh SK, Parker J, Ganesh NF, Wang PG, Gulati S, Rice PA: Implications of phase variation of a gene (pgtA) encoding a pilin galactosyl transferase in gonococcal pathogenesis. J Exp Med 2002, 196: 147-162.

3I. Jerse AE, Sharma ND, Simms AN, Crow ET, Snyder LA, Shafer WM: A gonococcal efflux pump system enhances bacterial survival in a female mouse model of genital tract infection. Infect Immun 2003, 71:5576-5582.

32. Rouquette-Loughlin CE, Balthazar JT, Hill SA, Shafer WM: Modulation of the mtrCDE-encoded efflux pump gene complex of Neisseria meningitidis due to a Correia element insertion sequence. Mol Microbiol 2004, 54:73I-74I.

33. Coen PG, Cartwright $K$, Stuart J: Mathematical modelling of infection and disease due to Neisseria meningitidis and Neisseria lactamica. Int J Epidemiol 2000, 29: 180-|88.

34. Snyder LAS, Bennett JS, Maiden MCJ, Saunders NJ: Two distinct gonococcal sub-populations cause invasive disease. . Unpublished manuscript

35. Bennett JS, Griffiths DT, McCarthy ND, Sleeman KL, Jolley KA, Crook DW, Maiden MC: Genetic diversity and carriage dynamics of Neisseria lactamica in infants. Infect Immun 2005, 73:2424-2432.

36. Parkhill J, Achtman M, James KD, Bentley SD, Churcher C, Klee SR, Morelli G, Basham D, Brown D, Chillingworth T, Davies RM, Davis P, Devlin K, Feltwell T, Hamlin N, Holroyd S, Jagels K, Leather S, Moule S, Mungall K, Quail MA, Rajandream MA, Rutherford KM, Simmonds M, Skelton J, Whitehead S, Spratt BG, Barrell BG: Complete DNA sequence of a serogroup $A$ strain of Neisseria meningitidis Z249 I. Nature 2000, 404:502-506.

37. Hamilton HL, Dominguez NM, Schwartz KJ, Hackett KT, Dillard JP: Neisseria gonorrhoeae secretes chromosomal DNA via a novel type IV secretion system. Mol Microbiol 2005, 55: $1704-172 \mid$.

38. McAllister CF, Stephens DS: Analysis in Neisseria meningitidis and other Neisseria species of genes homologous to the FKBP immunophilin family. Mol Microbiol 1993, 10:13-23.

39. Kellogg DSJ, Peacock WLJ, Deacon WE, Brown L, Pirkle Cl: Neisseria gonorrhoeae. I. Virulence genetically linked to clonal variation. Journal of Bacteriology 1963, 85: 1274-1279.

40. Bennett JS, Jolley K, Maiden MCI: Is the gonococcus a clonal complex of the meningococcus?: ; Oslo, Norway. Edited by: Caugant D and Wedege E. Nordberg Aksidenstrykkeri AS; 2002:42. 